\title{
Less Background, Better Contrast by Cooling Analyser Crystals
}

\author{
C.J. Carlile, M.A. Adams, P.S.R. Krishna, M. Prager $\ddagger$, \\ K. Shibata $\neq \ddagger$ and P. Westerhuijs \\ ISIS Pulsed Source, Rutherford Appleton Laboratory, \\ Chilton, Oxon OX11 0QX, UK
}

Cooling the pyrolytic graphite analyser crystals of the IRIS spectrometer to $25 \mathrm{~K}$ has proved to be very effective in suppressing the background from thermal diffuse scattering and in improving the contrast of the analysers. In this paper we show that a 12 times drop in background has been realised and that it would fall by an additional factor of 3-4 by further reducing the analyser temperature to $5 \mathrm{~K}$.

\section{Introduction}

Inelastic neutron spectroscopy on pulsed sources is carried out using two distinct methods: direct geometry spectrometers which use a monochromatic incident pulsed beam and detect energy changes at the sample by time of flight after scattering; and inverted geometry spectrometers which allow a white neutron beam to fall on the sample, energy sorted by time of arrival, which is then analysed after scattering at a single energy by an array of single crystals analysers.

Unlike equivalent direct-geometry instruments, inverted-geometry spectrometers can still be employed when carrying out measurements at high resolution and wide energy transfers from cold samples in their ground state. Using the inverted geometry neutron energy-loss technique the energy and momentum transfer ranges are largely independent from the energy resolution, which is a function of the selected analyser reflection energy and take-off angle.

Present addresses:

$\ddagger$ KFA Jülich, Germany

打 Tohoku University, Japan 
One reason why inverted-geometry spectrometers are a comparatively recent development is that it has generally been considered that the white beam falling on the sample in these spectrometers would cause more background than could be obtained with a direct-geometry machine. Indeed the neutron current passing through the sample is about one hundred times higher than on a similar performance directgeometry spectrometer. However, in recent years, spectrometers such as IRIS at ISIS and LAM80 at KEK Japan, following on from the success of the backscattering spectrometers at KFA Jülich and ILL, have demonstrated that the problem of the background resulting from the white beam in the spectrometer can be overcome quite satisfactorily.

Nevertheless white-beam techniques do mean that collimation of the incident and scattered beams must be very effective and that internal shielding must be welldefined and universal. A crucial factor in the sensitivity of such instruments is that the crystal used as the analyser must have a very high contrast for the analysing energy compared with all other energies in the incident beam. In practice this means that the coherent elastic cross section of the analyser must be very large compared to the sum of the coherent inelastic and the total incoherent cross sections.

For the IRIS spectrometer a 12 times drop in background has been achieved by cooling the pyrolytic graphite analyser crystals to $25 \mathrm{~K}$ and consequently reducing the coherent inelastic cross-section from thermal diffuse scattering (TDS) by this factor. Recent measurements have shown that the TDS will fall by a further factor of around 4 if the graphite were to be cooled to $5 \mathrm{~K}$. The overall gain in analyser contrast between room temperature and $5 \mathrm{~K}$ would then be close to a factor of 50 and the analyser contrast would approach 5000 . 


\section{Description of IRIS}

The IRIS spectrometer is illustrated in Figure 1. It consists of a long incident flight path from the liquid hydrogen moderator of the ISIS pulsed source which delivers a white pulsed neutron beam via a curved neutron guide to the sample sitting at $\mathbf{3 6 . 5}$ metres from the moderator. This flight path serves to time-sort the neutron beam such that the incident neutron energy is defined to microelectronvolt precision by time-offlight. The neutrons scattered from the sample are analysed by two large arrays of single crystals which reflect a single scattered neutron energy back to an array of scintillator detectors situated close to backscattering geometry $\left(\theta_{B}=87.5^{\circ}\right)$. The resolution of the primary spectrometer is then matched to that of the secondary spectrometer. Scattering angles from $15^{\circ}$ to $165^{\circ}$ are continuously covered with 51 scintillator detectors. Each analyser is 2.25 metres long, and $6 \mathrm{~cm}$ and $20 \mathrm{~cm}$ high respectively. The two sets of analysers on IRIS are pyrolytic graphite and muscovite mica. The resolution $\delta E$ of the instrument can be changed in steps by choosing the appropriate diffraction order of the analyser crystals: one obtains $\delta E=50 \mu \mathrm{eV}$ for PG $004,15 \mu \mathrm{eV}$ for PG 002, $4.5 \mu \mathrm{eV}$ for mica 004 and $1.1 \mu \mathrm{eV}$ for mica 002 . The former options select 3.3 and $6.6 \AA$ neutrons and the latter options utilise 9.9 and $19.8 \AA$ neutrons at the analyser. An important aspect of inverted geometry spectrometers is the ability to measure diffraction patterns simultaneously, thanks to the white incident beam. IRIS has a diffraction detector array situated at $2 \theta_{B}=170^{\circ}$ giving a resolution in $\Delta d / d$ of $2.510^{-3}$ over the d-spacing range from $2 \AA$ to $13 \AA$. The whole instrument is housed in a $2 \mathrm{~m}$ diameter stainless steel vacuum tank held at a pressure of $210^{-5}$ mbar. A fuller description of the IRIS spectrometer is given in Ref.[1]. 


\section{Cooling the analysers}

The analyser geometry of IRIS is very open as can be seen in Figure 1 - there is a line of sight between virtually each detector and each point on the analyser. A given detector is thus able to see a wide area of analyser surface whilst Bragg scattered neutrons are reflected to that detector from only a very limited area. The detector is therefore very sensitive to any non-specular scattering processes in the analysers. For graphite, since its incoherent cross-section is virtually zero (carbon is $98.9 \% \mathrm{C}^{12}$ ), these non-specular processes refer only to inelastic coherent scattering: thermal diffuse scattering from phonons. The contrast of the mica analyser on the other hand is determined by incoherent scattering since it contains a significant proportion of $\mathrm{OH}^{-}$ions and the contribution from TDS is relatively low.

As a consequence, the resolution function from the graphite analysers on IRIS at room temperature has the asymmetric shape characteristic of instruments on pulsed sources superimposed upon a broad almost flat underlying feature due to TDS. The ratio of elastic peak height to "flat" background with the analyser at room temperature is only 120 .

The origin of the TDS is illustrated in Fig. 2 where the scattered spectrum from a pyrolytic graphite analyser illuminated by a white beam is shown as a function of angular offset from the Bragg condition. These patterns were recorded in the IRIS diffraction detector with a graphite crystal at the sample position. As the crystal is turned off the Bragg condition, where the underlying TDS is centred on the intense diffraction line, two distinct 'wings' representing phonon creation and phonon annihilation emerge [2].

It is the sum of these offset patterns from a wide angular range of analysers which are recorded in each detector on the spectrometer and which results in the broad underlying feature centred on the elastic line. The Bragg line itself is reflected from only a restricted analyser area to each detector. The TDS intensity is approximately $1 \%$ of the elastic peak height, but the integrated intensity over the whole spectrum approaches that of the elastic line itself. One method of reducing the off-Bragg TDS intensity would be to introduce a coarse collimator between the analyser and the detector but this would leave unaffected the most intense contribution to the TDS signal which lies at or close to the Bragg reflection itself, and would significantly reduce the total signal. 
A second method, which is the solution taken on IRIS, is to cool the analyser to reduce the phonon population in the graphite and minimise the effects of TDS. Tests were carried out on IRIS with a graphite crystal in a helium cryostat at the sample position and scattering patterns were recorded in the diffraction detector as a function of offset from the Bragg condition at a series of temperatures down to $5 \mathrm{~K}$. In Fig. 3 the scattering patterns as a function of temperature are shown for an offset of $4^{\circ}$ from the $(002)$ reflection of graphite at a wavelength of $6.6 \AA$. The phonon annihilation and phonon creation peaks are clearly observed as is their drop with temperature.

In Fig. 4 the integrated TDS intensity for phonon annihilation and creation is shown to be almost linear with fall in temperature even down to $5 \mathrm{~K}$, because of the extremely soft phonon spectra which graphite possesses. The phonon energy of the TDS peaks observed as $\sim 2 \mathrm{meV}$ [4]. From these data, a graphite analyser operating at $25 \mathrm{~K}$ would be expected to produce a background from TDS sources which is more than an order of magnitude lower than it would be at room temperature. The elastic intensity itself hardly rises at all with the fall in temperature.

As a result of these tests the graphite analyser on IRIS has now been cooled to $25 \mathrm{~K}$, by a combination of cold compressed helium gas and liquid nitrogen cooling circuits, described in next section. The effect has been to improve the signal to background in the scattered spectrum (as defined by the peak intensity in the elastic line to the flat background from a $1 \mathrm{~mm}$ plate sample of vanadium) from 120-to-1 to 1350-to-1, more than a factor ten. This improvement in background is crucial in the reliable study of quasielastic neutron scattering (QENS ) and low intensity inelastic spectroscopy such as tunnelling. From Fig. 4 it can be seen that the background would fall a further factor of about 4 by reducing the analyser temperature to $5 \mathrm{~K}$. This is technically possible and a design is now being considered. 


\section{Technical Description of the Cooling System}

The graphite analyser comprises $13501 \mathrm{~cm}^{2}$ graphite pieces of grade ZYB $\left(\eta \sim 0.8^{\circ}\right)$ pyrolytic graphite supplied by Le Carbone of France. Each piece of $2 \mathrm{~mm}$ thickness is held to a single solid aluminium backing plate by miniature electronic plugs and sockets. The graphite is arranged in a $225 \times 6$ mosaic rectangular array, the overall length of the analysers on their backing plate being 2.30 metres. The front surface of the aluminium backing plate was cut by computer controlled machinery to the necessary quasi-spherical shape for focusing onto the detector bank. The analyser itself is supported by low conductivity convoluted stainless steel piping with a long heat path inside an aluminium radiation shield. A cross section of the analyser design is shown in Figure 5. The aluminium radiation shield is cooled to $80 \mathrm{~K}$ by flowing liquid nitrogen drawn through pipes by a gas pump. The analyser support plate itself is further cooled to $25 \mathrm{~K}$ by circulating helium gas at 20 bar which has been precooled by a closed-cycle Displex refrigerator. The front surface of the radiation shield is made from thin aluminium foil to minimise background scattering in the detectors and exposed parts of the analyser plate and shield are covered in $0.5 \mathrm{~mm}$ cadmium sheet. The temperature of the analysers and its shield is recorded by standard sensors and the position in time of flight of the elastic line itself acts as a monitor of the reducing $d$-spacing of the analyser with temperature.

\section{Conclusions}

The effectiveness of cooling the large graphite analyser on the IRIS spectrometer has been demonstrated. The analyser contrast of 120 at room temperature is improved to 1350 at $25 \mathrm{~K}$ by more than an order of magnitude reduction in TDS. Measurements show that further cooling the analysers to $5 \mathrm{~K}$ will increase this figure to beyond 5000. The energy gain process will fall almost to zero whereas energy loss processes will only drop marginally. At temperatures lower than $5 \mathrm{~K}$ other background effects will begin to become apparent at this level of sensitivity.

[1] C.J. Carlile and M.A. Adams, Physica B 182 (1992) 431

[2] B.T.M. Willis, C.J. Carlile, R.C. Ward, W.I.F. David and M.W. Johnson, Europhys. Lett., (1086) 2767

[3] E.A. Kellett and B.P. Richards, J. Appl. Cryst. (1971) 4, 1

[4] P.S.R. Krishna (private communication) 


\section{Figure Captions}

Figure 1: The IRIS high resolution spectrometer at the ISIS pulsed neutron source.

Figure 2: The scattering patterns from a single crystal of pyrolytic graphite measured by time of flight white beam diffraction at a series of offset angles from the Bragg condition. Peaks due to phonon creation at shorter times and phonon annihilation at longer times are clear as the offset angle increases.

Figure 3: Scattering patterns from pyrolytic graphite taken at a fixed offset angle from the Bragg condition of $4^{\circ}$ showing the reduction in intensity of the phonon peaks with fall in temperature. Structure caused by harder phonon branches can just be observed at the base of the remaining Bragg line in the centre of the pattern.

Figure 4: The integrated intensities of phonon creation and phonon annihilation peaks in graphite as a function of temperature.

Figure 5: A cross-section of the pyrolytic graphite analyser on IRIS showing the cooling mechanism and the analyser thermal shield and support. 


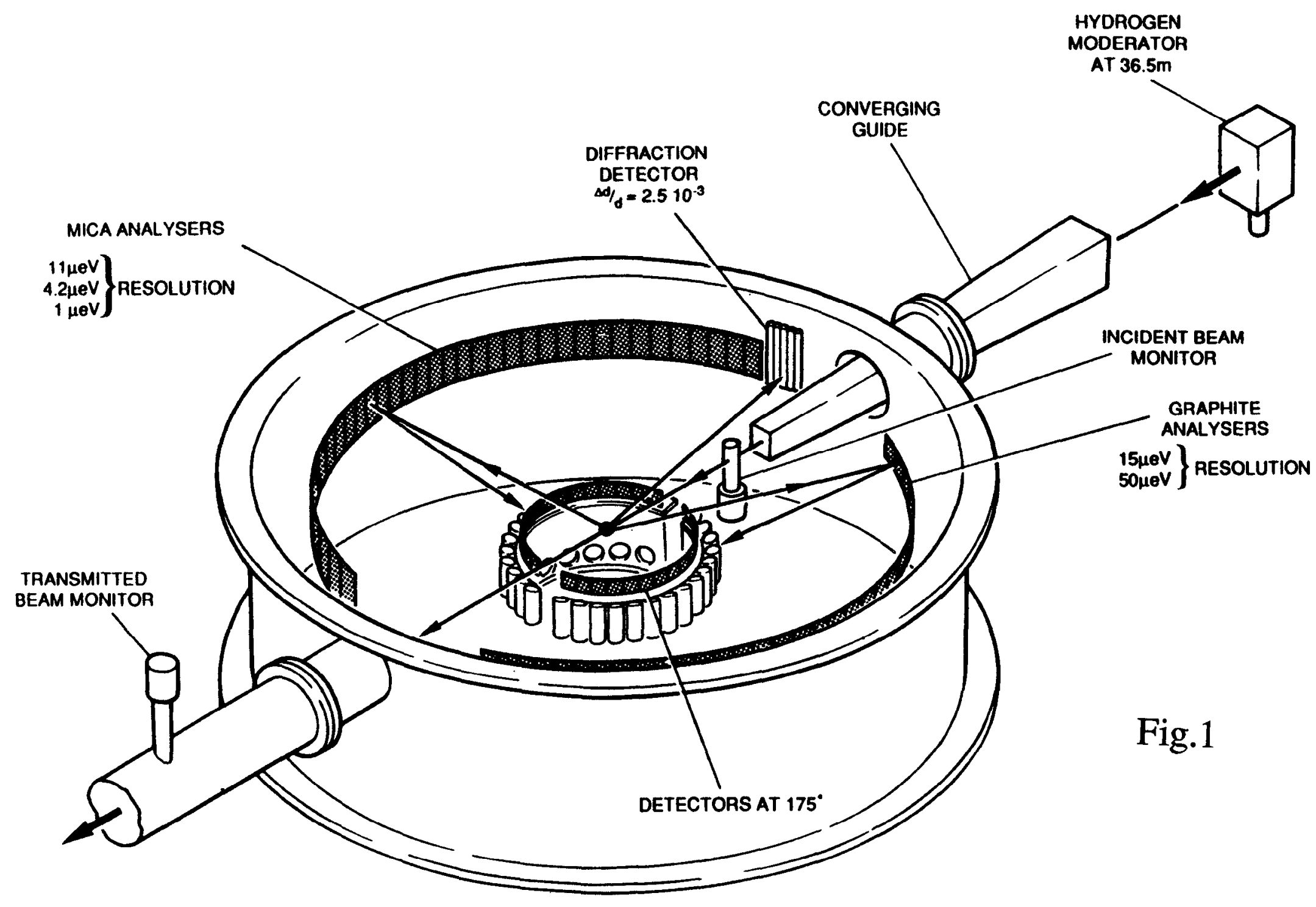

THE IRIS SPECTROMETER 


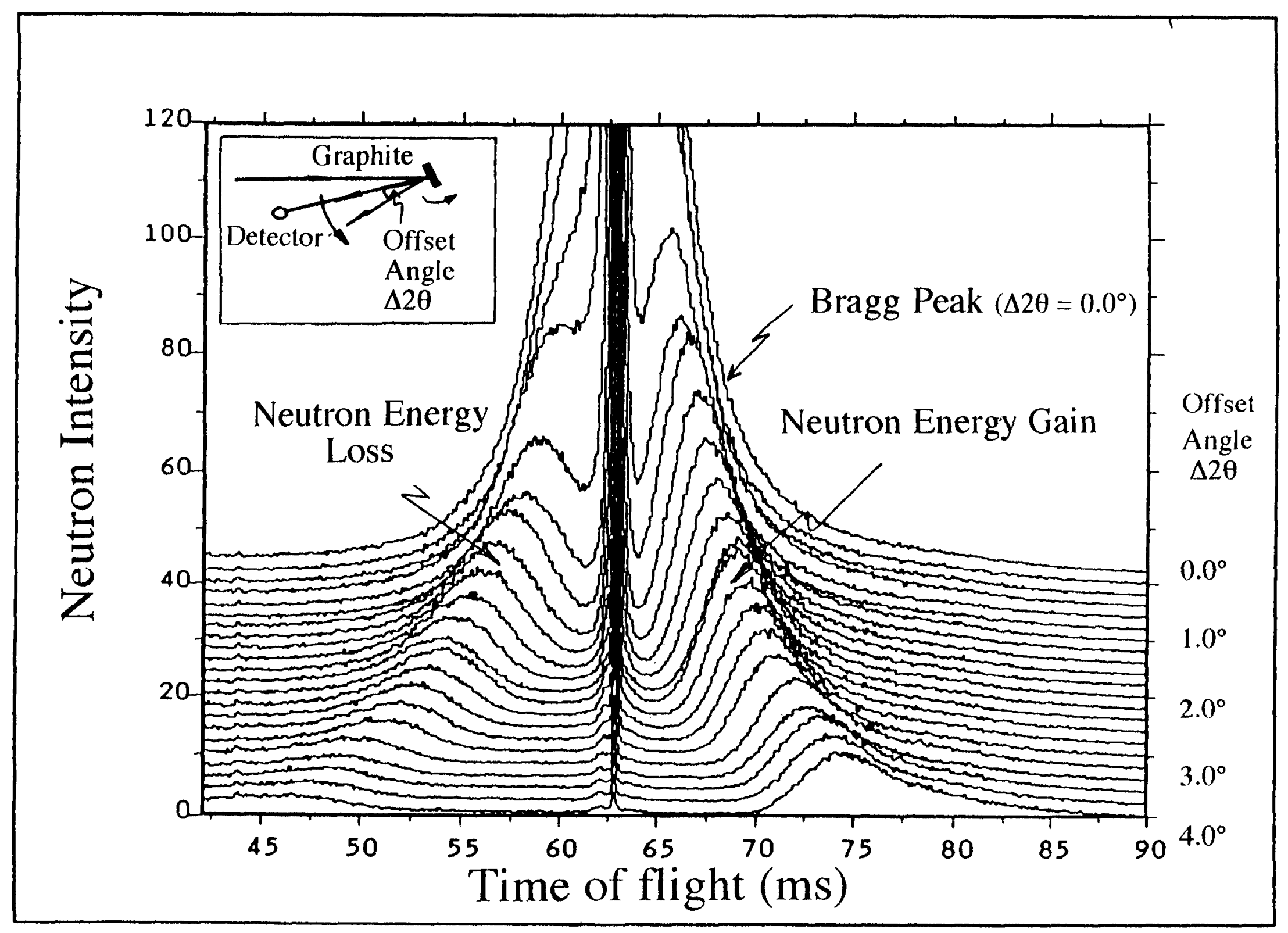

Fig.2 


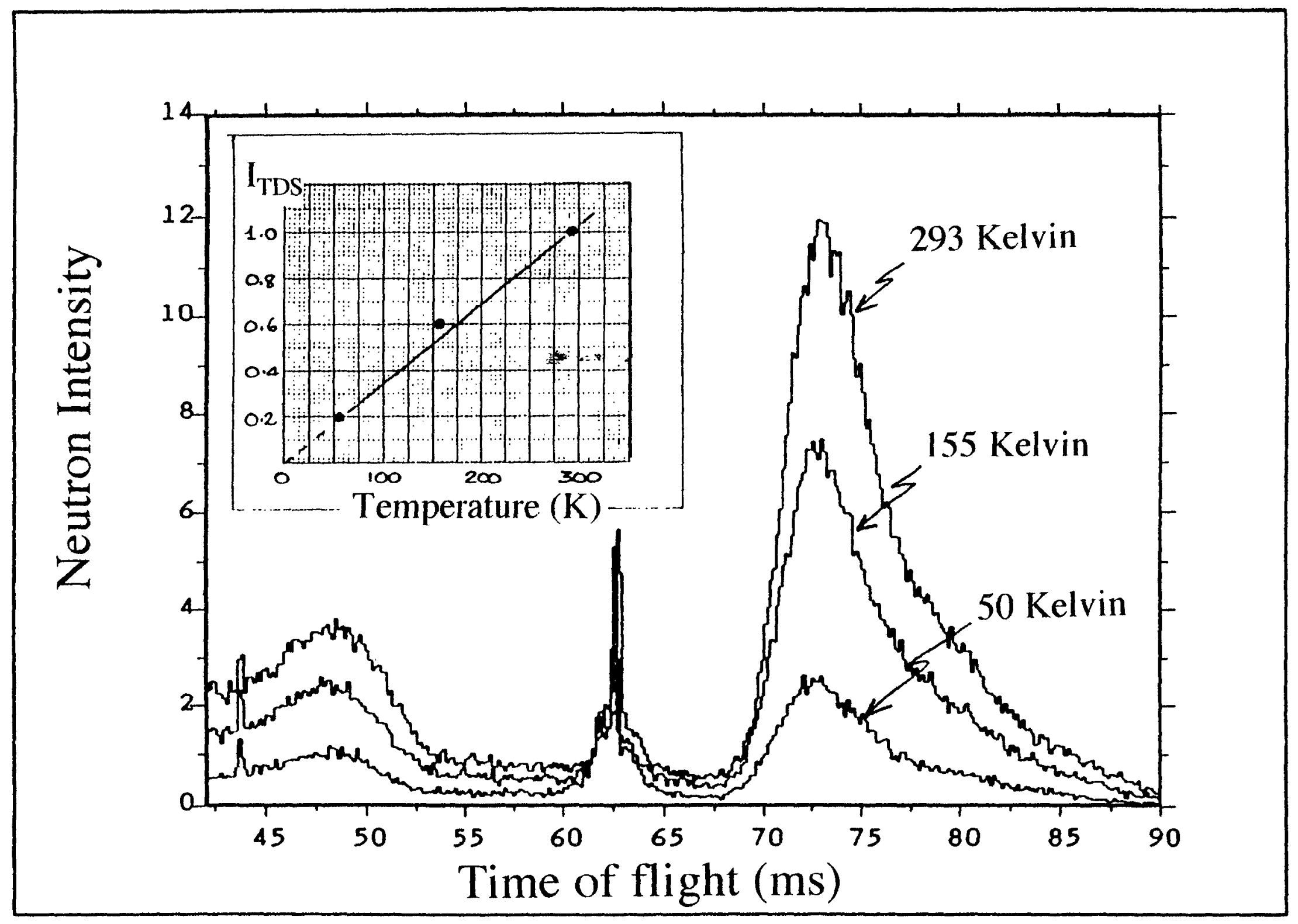

Fig.3 
Integrated Intensity (Arb. Units)

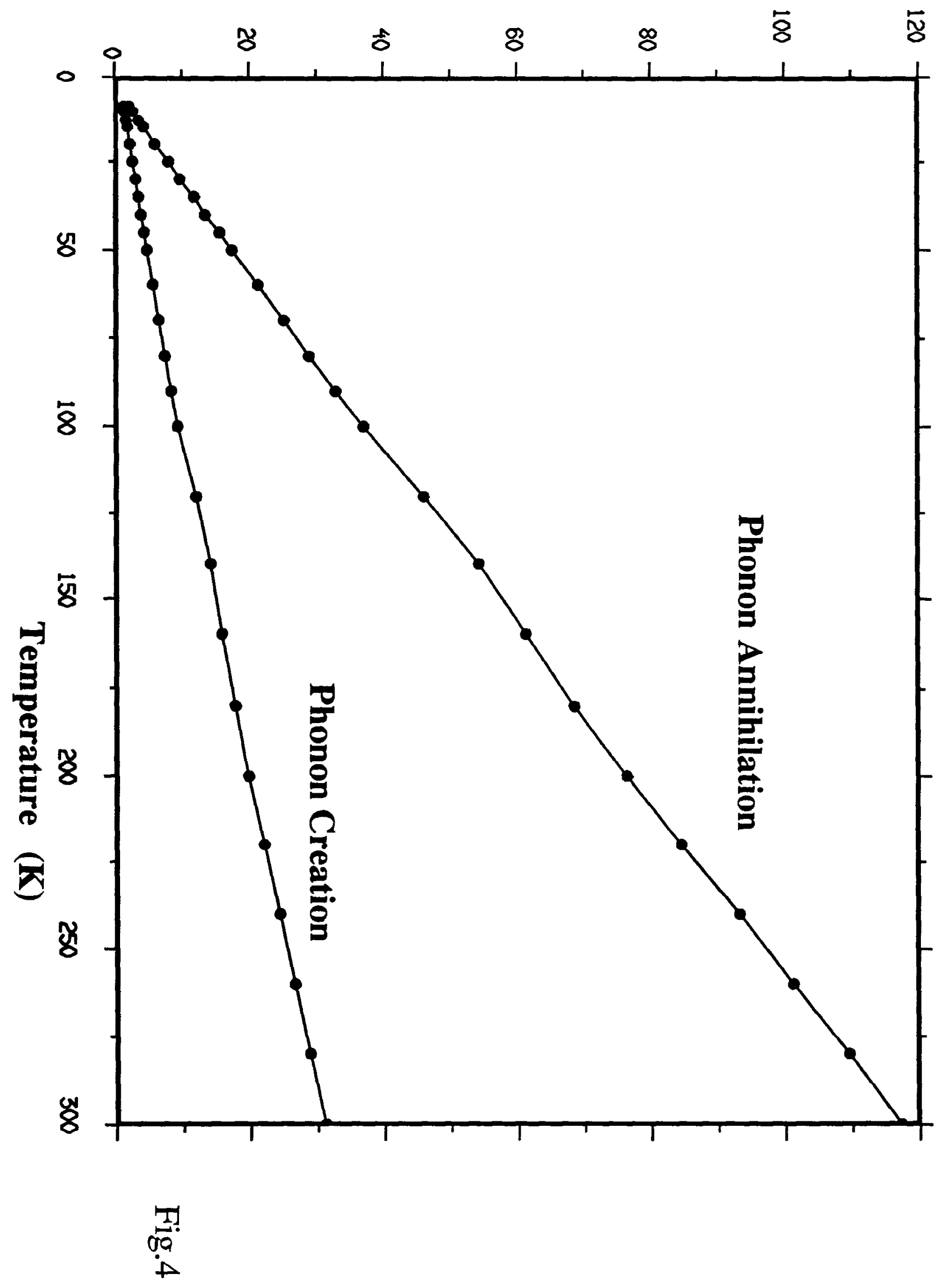




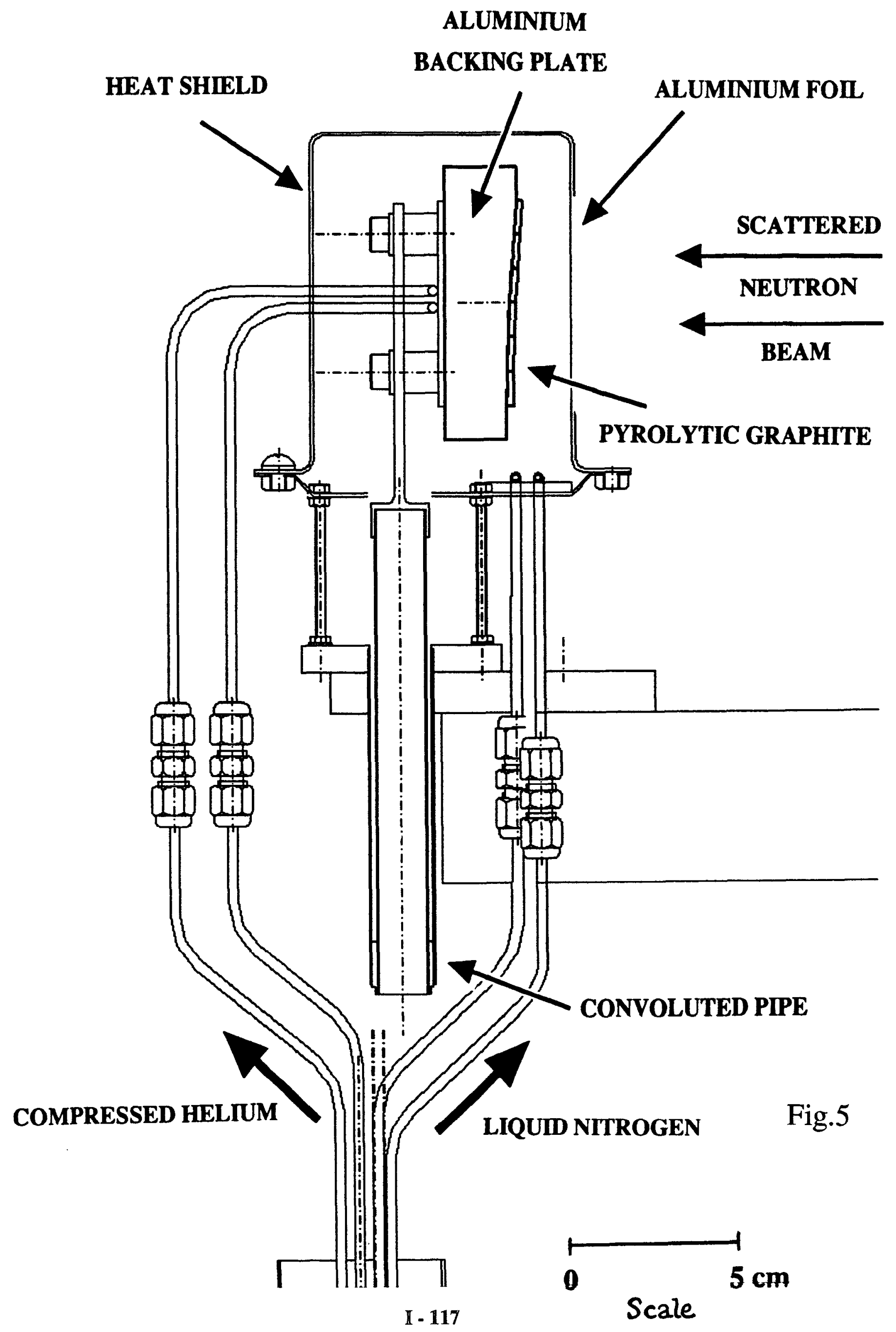

